\title{
Self-Assembly of Ordered Semiconductor Nanoholes by Ion Beam Sputtering
}

\author{
By Qiangmin Wei, Xiuli Zhou, Bhuwan Joshi, Yanbin Chen, Kun-Dar Li, \\ Qihuo Wei, Kai Sun, and Lumin Wang*
}

Low-energy-ion bombardment of semiconductors can lead to the development of complex and diverse nanostructures. Of particular interest in these structured surfaces is the formation of highly ordered patterns whose optical, electronic, and magnetic properties are different from those of bulk materials and might find technological uses. ${ }^{[1-5]}$ Compared to the low efficiency of lithographic methods for mass production, this self-organized approach offers a new route for fabrication of ordered patterns over large areas in a short processing time on the nanometer scale, beyond the limits of lithography. ${ }^{[1,4]}$ This technique is based on the morphological instability of a sputtered surface driven by a kinetic balance between roughening and smoothing. ${ }^{[6,7]}$ Thus mechanisms that control the species concentration on the surface can make contributions to structure formation..$^{[3,7-12]}$ It is now established that well-ordered quantum dots can be generated on the surface of semiconductors ( $\mathrm{Si}, \mathrm{Ge}, \mathrm{GaSb})$ under certain irradiation conditions. ${ }^{[1,13,14]}$ For a long time it has been expected that the instability of a surface can also lead to well-ordered hole formation. However, to date experimental observation of such features has been lacking. In this Communication, we report that a hexagonally ordered, honeycomb-like structure of holes $35 \mathrm{~nm}$ across and $45 \mathrm{~nm}$ apart on the Ge surface can be formed under focused ion beam (FIB) bombardment at normal incidence. The structured Ge fabricated by FIB bombardment shows a high surface area and a considerably blue-shifted energy gap. We found that interplay between ion sputtering, redeposition, viscous flow, and surface diffusion is responsible for ordered pattern formation. Simulations of the evolution of the surface morphology on the basis of the damped Kuramoto-Sivashinsky (DKS)

[*] Prof. L. M. Wang, Dr. Y. B. Chen, Dr. K.-D. Li

Department of Nuclear Engineering and Radiological Science University of Michigan

Ann Arbor, MI 48109 (USA)

E-mail: Imwang@umich.edu

Dr. Q. M. Wei, Dr. K. Sun

Department of Materials Science and Engineering

University of Michigan

Ann Arbor, MI 48109 (USA)

Prof. X. L. Zhou

School of Physical Electronics

University of Electronic Science and Technology of China Chengdu 610054 (P. R. China)

B. Joshi, Prof. Q. H. Wei

Department of Chemical Physics

Kent State University

Kent, $\mathrm{OH} 44242$ (USA)

DOI: 10.1002/adma.200803258 growth model have been performed to facilitate the interpretation of the experimental findings. ${ }^{[15-19]}$

As an indirect energy-gap semiconductor, germanium is a poor light emitter, which makes it challenging to create efficient Ge-based light-emitting devices. Significant effort has been devoted to the development of the optical properties of Ge based on changing the surface morphology. ${ }^{[20]}$ In the work reported here, we focused on the use of ion beam radiation to fabricate nanostructures on the Ge surface.

The ion-induced nanostructures were fabricated on commercially available Ge with (100) orientation by FIB bombardment. Under normal bombardment with ion energy greater than $5 \mathrm{keV}$, worm-like structures were developed on Ge surface with large aspect ratio. When the energy was $5 \mathrm{keV}$, however, highly ordered hole arrays could be achieved. Figure 1 shows scanning electron microscopy (SEM) and atomic force microscopy (AFM) images of a typical nanohole pattern induced on a $\mathrm{Ge}(100)$ surface by $5 \mathrm{keV}$ $\left(\mathrm{Ga}^{+}\right)$FIB bombardment for $5 \mathrm{~min}$. A perfect hexagonal arrangement of holes is observed within domains of ca. $500 \mathrm{~nm}$. Like polycrystalline structure, there are "grain boundaries" separating domains that are oriented randomly to each other by lattice defects. The mean diameter and the spacing of the holes measured from the SEM image (Fig. 1a) were 35 and $45 \mathrm{~nm}$, respectively. The high-magnification image in Figure $1 \mathrm{~b}$ shows the main defect types that interrupt the periodic arrangement of the holes. In order to identify substrate effects, we bombarded different-orientation samples and found that the ordering structure is orientation independent. The amorphous layer induced by the ion beam on the surface can lead to this substrate-independent ordering. ${ }^{[1,3,13,14]}$ Figure $1 c$ shows an AFM image obtained in tapping mode on a perfectly ordered domain, from which we find that there are hexagonally ordered quantum dots with $20 \mathrm{~nm}$ diameter and $3 \mathrm{~nm}$ height around each hole. The combination of well-ordered quantum dots and holes can be further confirmed by cross-sectional profiles (Fig. 1d) and three-dimensional (3D) structures (Fig. 1e). This honeycomb-like structure is reminiscent of the hexagonal structure in anodic alumina. $^{[21]}$

The dependence of holes on the bombardment time at a fixed energy of $5 \mathrm{keV}$ and flux of $2.2 \times 10^{15} \mathrm{~cm}^{-2} \mathrm{~s}^{-1}$ is shown in Figure 2 . At the very beginning ( $t=3 \mathrm{~s}$, Fig. $2 \mathrm{a})$, corresponding to an ion fluence of $6.6 \times 10^{15} \mathrm{~cm}^{-2}$, hole nucleation occurs. The network structure with broad hole-size distribution can be observed, suggesting random nucleation sites. The hole formation can be attributed to the aggregation of vacancies on the surface generated by energetic ion sputtering. As bombardment proceeds ( $t=30 \mathrm{~s}$, Fig. $2 \mathrm{~b}$ ), more surface Ge atoms are removed and visible short-range ordering of holes can be observed within 

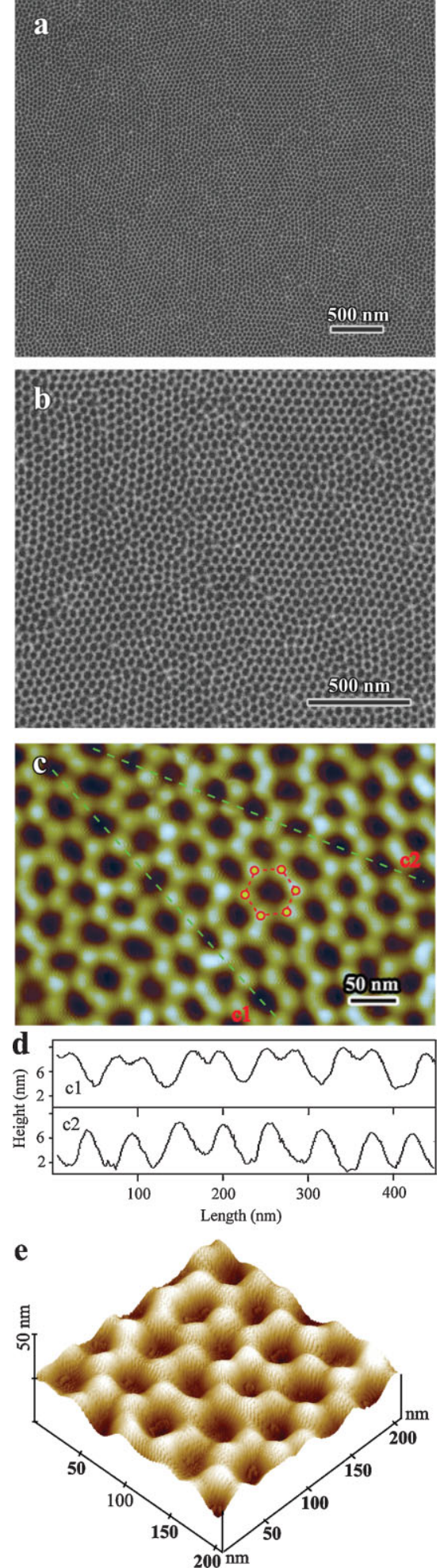

Figure 1. a,b) SEM images of hexagonally ordered nanoholes on a Ge surface at low (a) and high (b) magnification. c) AFM image of a hexagonally ordered hole domain showing a perfect hexagonal arrangement of bumps around each hole (indicated by circles and connecting lines). d) Cross-sectional profile corresponding to the dashed lines $\mathrm{Cl}$ (top) and c2 (bottom) marked in (c). e) 3D image of ordered pattern. Ion energy $5 \mathrm{keV}$, flux $2.2 \times 10^{15} \mathrm{~cm}^{-2} \mathrm{~s}^{-1}$, bombardment time $5 \mathrm{~min}$.
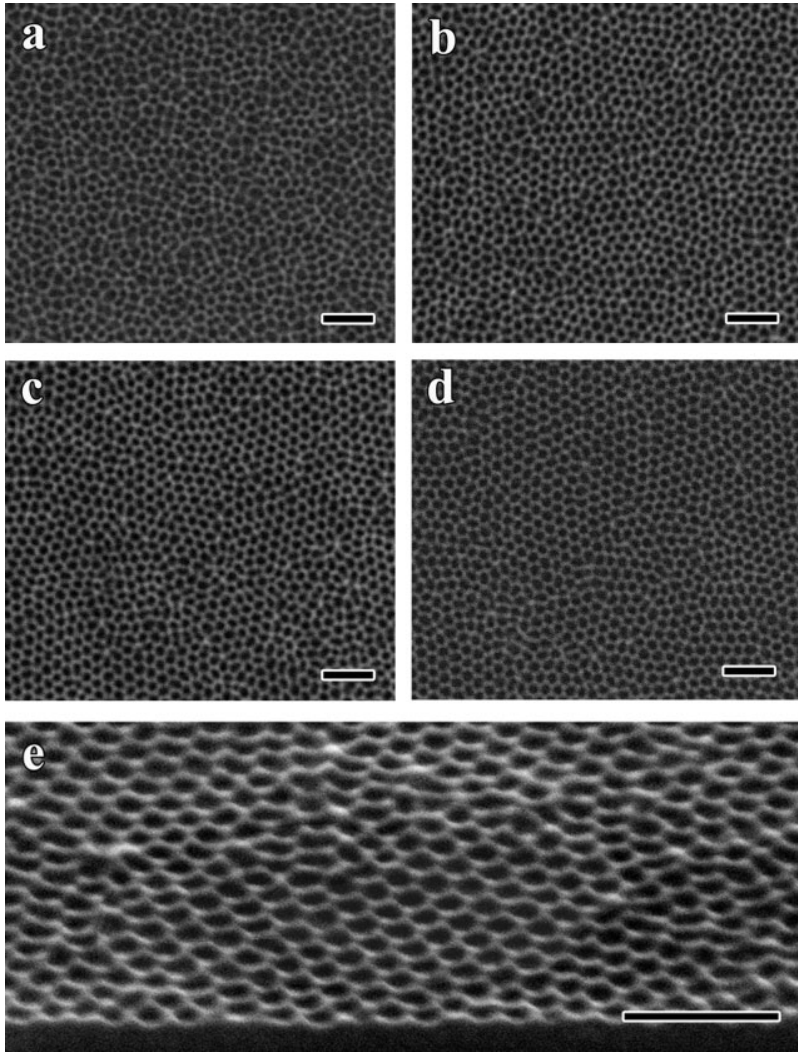

Figure 2. SEM images showing evolution of Ge surface at different times: a) $3 \mathrm{~s}$, b) $30 \mathrm{~s}$, c) $90 \mathrm{~s}$, and d) $240 \mathrm{~s}$. e) Cross-sectional SEM image of (c) viewed from $52^{\circ}$ to normal. lon energy $5 \mathrm{keV}$, flux $2.2 \times 10^{15} \mathrm{~cm}^{-2} \mathrm{~s}^{-1}$, scale bar $200 \mathrm{~nm}$

small domains. For longer bombardment times (Figs. 2c and d), the hole size becomes uniform by self-adjusting and the ordered domain slowly increases in size. A cross-sectional SEM image (Fig. 2e) shows the bowl-like shape of the holes with a depth of $5 \mathrm{~nm}$.

Structural and chemical characterization of the nanoholes induced by the ion beam is shown in Figure 3. The nanohole structure can be created on a thin sample that can be traversed by the electron beam. A plan-view transmission electron microscopy (TEM) bright-field image (Fig. 3a) shows the surface structure of Ge induced by a $5 \mathrm{keV}$ FIB before and after annealing. The thickness variation produces different contrast, in which the holes are brighter. The cross-sectional TEM image shows a dot-shaped structure with a depth of $5 \mathrm{~nm}$ and sidewall angle of $60^{\circ}$ to $70^{\circ}$ (Fig. 3b). An amorphous layer approximately $5 \mathrm{~nm}$ thick is produced by energetic ions on the surface. This layer, consistent with the penetration depth of the ions calculated by Monte Carlo simulation, ${ }^{[22]}$ remains constant during bombardment. Figure 3c shows a high-angle annular dark field (HAADF) TEM image, in which the holes are darker. The element mapping obtained by scanning transmission electron microscopy (STEM) shows uniform distribution of implanted $\mathrm{Ga}$ inside the $\mathrm{Ge}$ substrate. At bombardment temperature, no solid solution or compound between $\mathrm{Ga}$ and $\mathrm{Ge}$ can be found. Annealing at $600^{\circ} \mathrm{C}$ 

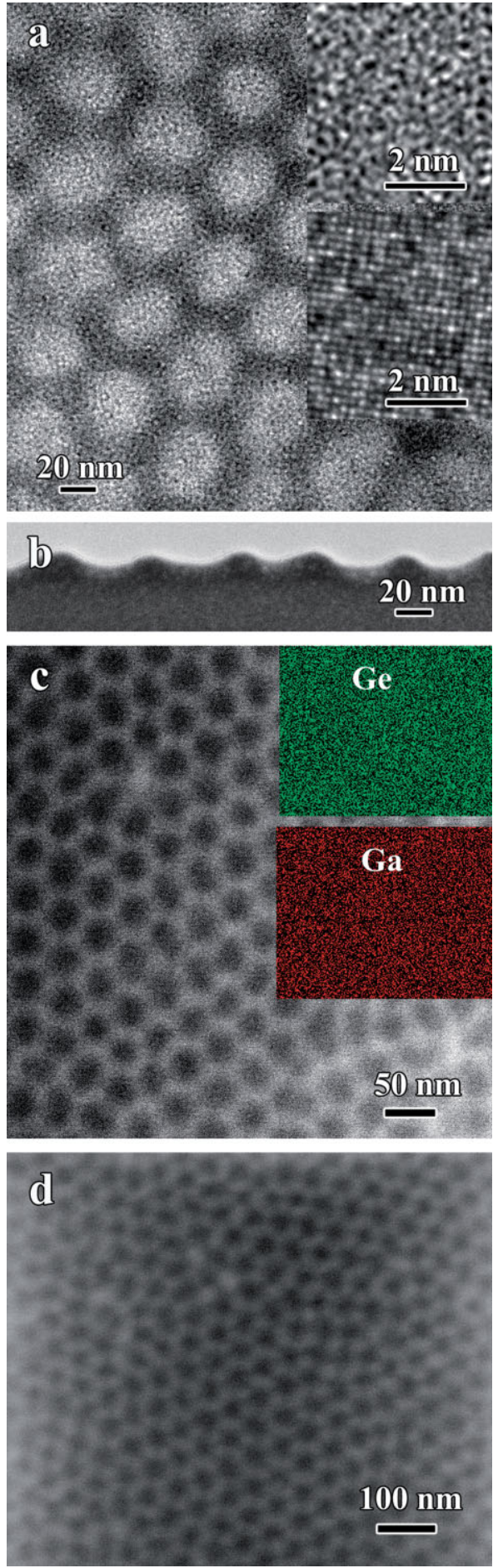

Figure 3. Structural and chemical characterization of nanoholes on a Ge surface. a) Plan-view TEM image of surface morphology on a thin Ge foil. Insets: High-resolution TEM images of sample before (top) and after (bottom) annealing. b) Cross-sectional TEM image of the nanohole structures. c) HAADF image. The contrast variation is a result of height modulation: holes are darker. Insets: EDXS mapping acquired by STEM, where the distributions of $\mathrm{Ge}$ (top) and $\mathrm{Ga}$ (bottom) atoms are given. d) SEM image showing hexagonally ordered nanoholes on Ge surface after annealing at $600^{\circ} \mathrm{C}$ for half an hour. The pattern was generated by the same irradiation conditions as in Figure 1. for half an hour leaves the hexagonal arrangement of the holes undamaged, as shown in Figure 3d.

The mechanism underlying pattern formation during ion bombardment has been extensively investigated in recent years. Under ion bombardment, two main defects, vacancies and interstitials, are created with the decrease of ion energy. Atoms on the surface can also be sputtered, leaving vacancies on the surface. Some of these sputtered atoms can redeposit on the surface to take part in structure formation. In addition, amorphous layer can be generated on the semiconductor surface and thus viscous flow should be considered to describe the behavior of this layer. Furthermore, thermally induced instability of defects also plays an important role in pattern formation. As a consequence, to date four main mechanisms have been considered: sputtering, surface diffusion, redeposition, and viscous flow. ${ }^{[0,7,10,11]}$ Because all these effects are surface profile dependent, for simplicity, a small slope approximation was proposed, and thus a linear continuum equation was established to explain the morphology evolution at the very beginning. ${ }^{[6]}$ As bombardment proceeds, nonlinear effects start to dominate the surface dynamics and a nonlinear equation was developed by considering surface profile changes. ${ }^{[7,10,12]}$ It was found that the DKS growth model can describe well these four mechanisms for the long-time limit. ${ }^{[18,19,23,24]}$ According to this model, a positive nonlinear term describes dot formation and a negative nonlinear term predicts hole formation. It has been reported that the DKS growth model can successfully predict ordered quantum dot formation during bombardment with a positive sign of the nonlinear term. ${ }^{[18]}$ The DKS growth model is given by

$\frac{\partial h}{\partial t}=-\left(\alpha+\nu \nabla^{2}+D \nabla^{4}\right) h-\lambda(\nabla h)^{2}+\eta$

where $h$ is the height of the bombarded surface as a function of time $t, v$ is effective surface tension generated by the erosion process due to surface curvature or viscous flow due to surface stress, $D$ is effective diffusion coefficient from thermal diffusion, radiation induced diffusion, and viscous flow, $\lambda$ describes the tilt-dependent sputtering yield, determining the dot or hole formation, $\eta$ is Gaussian white noise resulting from the stochastic nature of the erosion process, and a damping term $-\alpha h$ accounts for the redeposition of sputtered species on the surface. The value of $\lambda$ can be calculated from parameters for ion energy range distribution. ${ }^{[7]}$ In our case, we found $\lambda$ is positive, which means hole formation can be predicted from the DKS model. Under normal bombardment, by rescaling coefficients, Equation 1 yields an isotropic partial differential equation with $v=D=\lambda=1$. $^{[23]}$ Therefore, for the isotropic DSK model, the variation of $\alpha$ determines the morphology evolution.

Numerical simulation has been performed on an equally spaced 2D $512 \times 512$ mesh by integration of Equation 1 using a standard discretization method with periodic boundary conditions. The integration starts from a random initial state with spatial step $\mathrm{d} x=0.5$, time step $\mathrm{d} t=0.005, v=D=\lambda=1, \eta=0$, and $\alpha=0.23$. When the iteration is less than $1 \times 10^{5}$, randomly distributed holes were developed. The ordered hole patterns start to form around iterations of $2 \times 10^{5}$, a long-time limit where nonlinear effects are relevant. Figure 4 shows a comparison of 

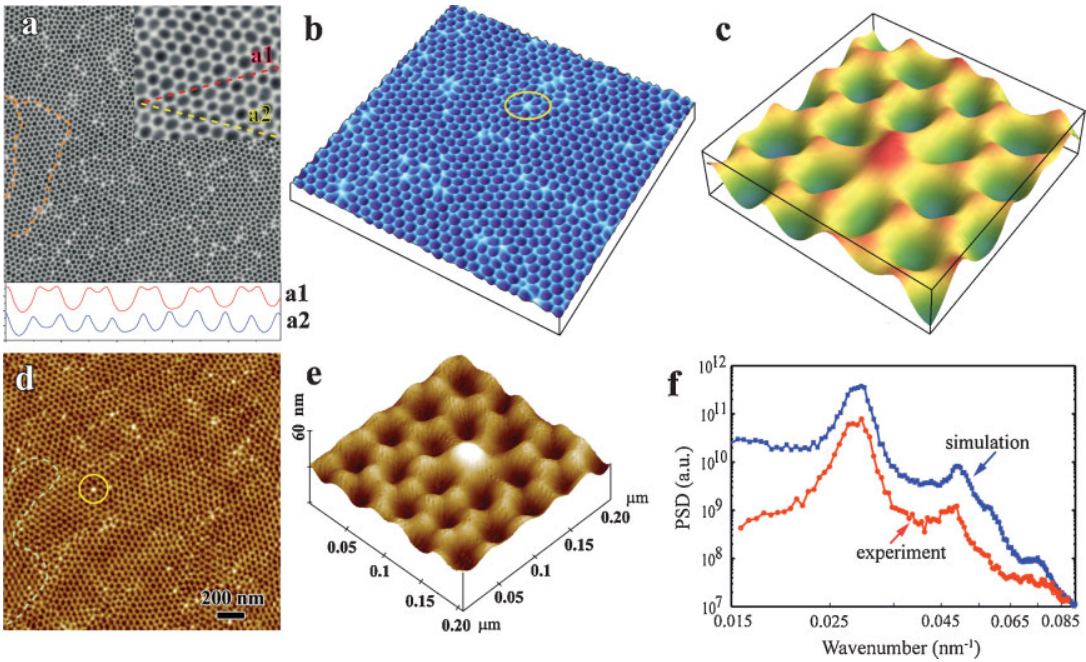

Figure 4. Comparison of surface morphology calculated by DKS equation with experimental observations. a) Numerical simulation from Equation 1 with $\alpha=0.23$ at $t=2000\left(4 \times 10^{5}\right.$ iterations). Inset: High-magnification image of an ordered hole domain and corresponding cross sections of surface topography. b) 3D structure of numerical results. A point defect (a dot instead of a hole surrounded by six nearest holes) is circled. c) Enlarged segment of defect structure from the area circled in (b). d) AFM image of ordered patterns on Ge surface created by $5 \mathrm{keV}$ ions for fluence of $6.6 \times 10^{17} \mathrm{~cm}^{-2}$. A point defect is circled. e) Enlarged AFM image showing point defect circled in (d). f) Log-log plot of the PSD curves obtained from (a) and (d). Dashed curves in (a) and (d) show line defects, which separate the domains.

predictions of Equation 1 with experimental observations. Clearly, a striking similarity of surface morphology between simulation results and experiment can be found from 2D images (Figs. 4a,d) and 3D images (Figs. 4b,c,e). In particular, the perfect hexagonal arrangement of holes, domains, and grain-like boundaries predicted by Equation 1 matches very well with experimental observations. The ordering of patterns can be well characterized by the power spectral density (PSD) calculated from the Fourier transform of the intensity of the images. The angularly averaged PSD calculated from simulation (Fig. 4a) is again in good agreement with that from experimental data obtained by AFM measurement (Fig. 4d). Similar to experimental observations, hexagonally ordered dots around each hole can also be predicted by simulation, as can be seen in the cross-sectional profile in Figure $4 \mathrm{a}$ and 3D structure in Figures $4 \mathrm{~b}$ and c. These results
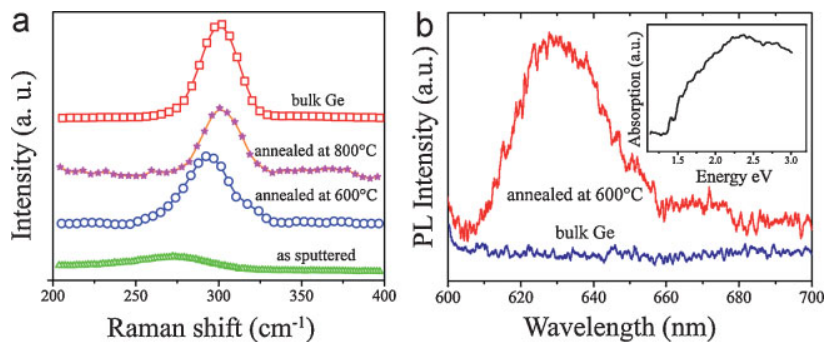

Figure 5. Optical properties of ion-etched Ge. a) Raman spectra of samples annealed at different temperatures. b) Room temperature photoluminescence $(\mathrm{PL})$ spectra of $\mathrm{Ge}$ patterned with ordered nanoholes after annealing at $600^{\circ} \mathrm{C}$. The spectrum of bulk $\mathrm{Ge}$ is given for comparison. Inset: Absorption spectrum of patterned Ge after annealing at $600^{\circ} \mathrm{C}$. A $530 \mathrm{~nm}$ Ar ion laser was used for luminescence excitation. confirm that the DKS growth model can fairly well explain hole formation where nonlinear effects are prerequisite for ordering.

Visible light emission from semiconductor nanostructures has attracted significant attention because of potential optoelectronic applications. Figure 5a shows the Raman spectrum of an optical phonon near $300 \mathrm{~cm}^{-1}$ wave number excited by a $530 \mathrm{~nm}$ laser after annealing. For an unannealed sample, similar to amorphous structure, the Raman spectrum shows a broad bump around $275 \mathrm{~cm}^{-1}$. With increasing annealing temperature, a sharp peak can be identified and shifts to the position of bulk Ge, suggesting well-crystallized Ge. The photoluminescence (PL) spectrum of ion-etched Ge after annealing at $600{ }^{\circ} \mathrm{C}$ (Fig. 5b) shows a broad but pronounced peak ranging from $500 \mathrm{~nm}$ to $700 \mathrm{~nm}$. No weak peak can be observed for the unannealed sample. The optical absorption spectrum of ion-etched $\mathrm{Ge}$ after annealing at $600^{\circ} \mathrm{C}$ shows a blue shift in the energy gap transition from $0.66 \mathrm{eV}$ to $1.42 \mathrm{eV}$, compared with crystalline or amorphous bulk Ge. This bandgap change can be attributed to the quantum confinement resulting from the dimensional reduction of the hole wall thickness. ${ }^{[18,20]}$

In conclusion, we have shown that, in contrast to well-ordered quantum dot formation in most ion sputtered semiconductors, highly ordered nanoholes can be spontaneously generated on the surface of Ge under certain irradiation conditions. The formation and evolution of nanoholes are determined by the concentration and mobility of species induced by the ion bombardment on the surface. Many mechanisms are involved in this pattern formation, which provides tremendous opportunities for the fabrication of nanostructures with different shapes and functions. The observed features are in good agreement with those predicted in numerical simulations for the DKS growth model under the conditions of long-time limit, implying the importance of nonlinear effects in the pattern formation.

\section{Experimental}

Commercially available Ge with (100) orientation was used in our experiments (undoped $\mathrm{n}$-type from $\mathrm{mTI}$ Corporation). The ion bombardment experiments were carried out using a FIB instrument (FEI Nova 200 Nanolab, $\mathrm{Ga}^{+}$ion) equipped with a scanning electron microscope in a vacuum of $2 \times 10^{-7} \mathrm{mbar}$ at room temperature. The spot size of the $5 \mathrm{keV}$ $\mathrm{Ga}^{+}$ion beam was $50 \mathrm{~nm}$ with an overlap of $50 \%$. Each spot was bombarded for $1 \mu \mathrm{s}$ with a repetition time of $100 \mathrm{~ms}$. The surface morphology was characterized by in situ SEM and ex situ TEM and AFM. AFM measurements were carried out in tapping mode under ambient conditions using phosphorus-doped Si cantilevers (Nanoscope IV). Cross-sectional TEM samples were prepared by FIB and analyzed by TEM (JEOL 2010 F) with a field emission gun operated at $200 \mathrm{keV}$. The chemical composition was analyzed by energy dispersive X-ray spectrometry (EDXS) and mapping of electron energy-loss spectroscopy. Fast Fourier transforms (FFTs) and PSDs were calculated to identify the ordering. 


\section{Acknowledgements}

This work was supported by the Office of Basic Energy Science of the U.S. Department of Energy through grant no. DE-FG02-02ER46005.

Received: November 5, 2008 Revised: March 9, 2009 Published online: April 20, 2009

[1] S. Facsko, T. Dekorsy, C. Koerdt, C. Yrappe, H. Kurz, A. Vogt, H. L. Hartnagel, Science 1999, 285, 1551.

[2] B. Ziberi, F. Frost, M. Tartz, H. Neumann, B. Rauschenbach, Appl. Phys. Lett. 2008, 92, 063102.

[3] Q. M. Wei, J. Lian, W. Lu, L. M. Wang, Phys. Rev. Lett. 2008, 100, 076103.

[4] R. Gago, L. Vázquez, O. Plantevin, T. H. Metzger, J. Muñoz-García, R. Cuerno, M. Castro, Appl. Phys. Lett. 2006, 89, 233101.

[5] A.-D. Brown, J. Erlebacher, W. L. Chan, E. Chason, Phys. Rev. Lett. 2005, 95, 056101.

[6] R. Bradly, J. Harper, J. Vac. Sci. Technol. A 1988, 6, 2390.

[7] R. Cuerno, A.-L. Barabási, Phys. Rev. Lett. 1995, 74, 4746.

[8] J. Erlebacher, M. J. Aziz, E. Chason, M. B. Sinclair, J. A. Floro, Phys. Rev. Lett. $1999,82,2330$
[9] Q. M. Wei, W. X. Li, K. Sun, J. Lian, L. M. Wang, J. Appl. Phys. 2008, 103, 074306.

[10] M. Castro, R. Cuerno, L. Vázquez, R. Gago, Phys. Rev. Lett. 2005, 94, 016102.

[11] P. F. A. Alkemade, Phys. Rev. Lett. 2006, 96, 107602.

[12] J. Muñoz-García, M. Castro, R. Cuerno, Phys. Rev. Lett. 2006, 96, 086101.

[13] R. Gago, R. Cuerno, M. Varela, C. Ballesteros, J. M. Albella, Appl. Phys. Lett. 2001, 78, 3316.

[14] B. Ziberi, F. Frost, B. Rauschenbach, Appl. Phys. Lett. 2006, 88, 173115.

[15] R. Gago, L. Vázquez, O. Plantevin, J. A. Sánchez-García, M. Varela, M. C. Ballesteros, J. M. Albela, T. H. Metzger, Phys. Rev. B 2006, 73, 155414.

[16] F. Frost, A. Schindler, F. Bigl, Phys. Rev. Lett. 2000, 85, 4116.

[17] A. Pascale, I. Berbezier, A. Ronda, P. C. Kelires, Phys. Rev. B 2008, 77, 075311.

[18] S. Facsko, T. Bobek, A. Stahl, H. Kurz, T. Dekorsy, Phys. Rev. B 2004, 69, 153412.

[19] B. Kahng, H. Jeong, A.-L. Barabási, Appl. Phys. Lett. 2001, 78, 805.

[20] G. S. Armatas, M. G. Kanatzidis, Science 2006, 313, 817.

[21] F. Y. Li, L. Zhang, R. M. Metzger, Chem. Mater. 1998, 10, 2470.

[22] J. F. Ziegler, J. P. Biersack, U. Littmark, The Stopping and Range of lons in Solids, Pergamon, New York 1985.

[23] S. Vogel, S. J. Linz, Europhys. Lett. 2006, 75, 884.

[24] M. Paniconi, K. R. Elder, Phys. Rev. E 1997, 56, 2713 